\title{
Prevalence of Antimicrobial Resistance Patterns of Escherichia coli Faecal Isolates of Cattle
}

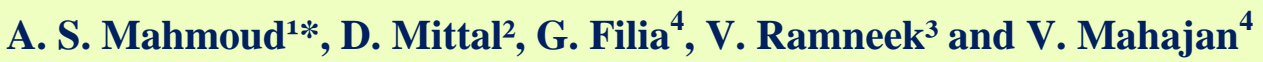 \\ ${ }^{1}$ Department of Preventive Medicine, Faculty Veterinary Medicine, University of Tripoli, \\ Tripoli, Libya \\ ${ }^{2}$ Department of Veterinary Public Health and Epidemiology, LalaLajpatRai University of \\ Veterinary \& Animal Sciences, Hisar-125004 (Haryana) India \\ ${ }^{3}$ Department of Animal Biotechnology, School of Animal Biotechnology, GADVASU, \\ Ludhiana \\ ${ }^{4}$ Animal Disease Research Centre, GADVASU, Ludhiana, India, India \\ *Corresponding author
}

Keywords

E. coli, Antibiotics resistance,

Multiplex PCR, tet genes, Risk factors

Article Info

Accepted:

12 February 2020

Available Online:

10 March 2020

\section{A B S T R A C T}

A total of 154 of Escherichia coli (E. coli) isolates were isolated from faecal specimens of healthy cattle $(\mathrm{n}=100)$. Serotyping of $154 \mathrm{E}$. coli isolates indicated that $112(72.72 \%)$ isolates were typeable, $28(18.18 \%)$ untypeable and $14(9.09 \%)$ were rough. The most predominant serotype observed was $\mathrm{O} 22$ followed by O56, O60, O120 and O1. Antibiogram pattern using a total of 25 different antibiotics indicated that high resistant was present against clindamycin, metronidazole and penicillin followed by cephalothin, neomycin, kanamycin, cephalexin, streptomycin, furazolidone and tetracycline antibiotics. The amplification of tet(A) and tet(B) resistant determinants by PCR resulted in generation of $372 \mathrm{bp}$ and 228 , respectively. The tet(A) gene was predominant gene compared to tet(B) gene. This study using pheno-genotypic characterization indicated the presence of antimicrobial resistant $E$. coliisolates amongst healthy cattle, emphasizing that effective strategy should be applied to persist the efficiency along with ideal usage of novel antibiotics though minimizing the risk of antibiotic resistant bacteria.

\section{Introduction}

Antibiotic resistance is of great concern and various strategies have been conducted for its monitoring and investigation in the veterinary practices and public health. Antimicrobials are used frequently for curing, controlling and prophylactic of various transmissible diseases 
in animals and humans. To successfully control infectious diseases of bovines and to prevent the possible hazard linked with bacterial resistance and therapeutic failure, it is essential to evaluate antibiotic resistance of pathogenic bacteria (Authier et al., 2006). The improper and extensive use of antibiotic in veterinary practices is undoubtedly the main cause of bacterial resistance (Sorum and Sunde, 2001)

The intestinal tract of the animals and humans is considered as a main reservoir for the commensal bacteria, though $E$. coli represents one of the most significant bacterial commensal in the intestinal tract of animals and humans (Rosas et al., 2006). However, some serotypes are able to causes illness in both humans and animals, like, haemorrhagic colitis, urinary tract infections (UTIs). The resistance of the E. coli against commonly practised antimicrobials in veterinary and human medicine has progressively increased. Amongst resistance to many antimicrobial agents, the resistant to tetracycline is commonly reported in zoonotic, pathogenic and non-pathogenic bacteria like $E$. coli (Authier et al., 2006). The tetracyclines are widely used in animals and humans due to its broad spectrum activity and therapeutic efficacy with low side effects. The mechanism for resistance to tetracycline can be classified into four categories and 35 different tetracycline or oxytetracycline genes are involved (Chopra and Roberts, 2001).

Scientist are nowadays concerned on therapeutic usage of antimicrobial agents and concurrent development of antibiotic resistant pathogenic bacteria that might be of slipover to humans either through dealings with food, animal foodstuff chain and/or through food producing animals (Lanz et al., 2003). However, the uses of antibiotics in various veterinary fields may potentially influence the antimicrobial resistance profile in both exogenous infectious bacteria and commensal bacteria like E. coli (Catry et al., 2003).

Despite withdrawal of antimicrobial agent from the rations, the bacterial resistant can be still determined for a long time (Langlois et al., 1988; Gellin et al., 1989) in animals. Therefore, a commensal bacteria that poses antimicrobial resistance determinants constitutes a main resource of resistant genes for highly pathogenic bacteria (Moyaert et al., 2006). The studies involved in the antimicrobial resistance are frequently targeted to assessing resistant phenotypes that might originate from diverse genetic determinants, however, these determinants might represent a particular epidemiological pattern. Consequently, the genotypic assessment of antibiotic resistance is considered as significant for monitoring antimicrobial resistant diseases.

\section{Materials and Methods}

\section{Samples collection}

A total of hundred faecal specimens $(n=100)$ were collected aseptically and randomly from apparently healthy cattle of varying age groups located in different agro-climatic zones of the Punjab state, India. The faecal samples were collected into small sterile plastic bag wearing sterile gloves directly from animals per rectal and processed directly in the laboratory for further microbiological investigation.

Isolation, serotyping and phenotypic antibiotic resistant profile of the $E$. coli isolates

MacConkey lactose bile salt agar (MLA, HiMedia) was used for initial isolation. The faecal samples were streaked on freshly prepared MLA and incubated at $37^{\circ} \mathrm{C}$ for 24 hours. Preliminary identification was carried 
out on the basis of Gram reaction, morphology and colony characteristics. Subsequently, pure E. coli colonies were analysed by biochemical tests for confirmation as described by Quinn et al., (1994). The confirmed pure isolates were stored at $4^{\circ} \mathrm{C}$ using tryptone soya agar slants. The serotyping of E. coli isolates was carried out at the Central Research Institute, Kasauli, Himachal Pradesh, India. The phenotypic antibiotics sensitivity of the $E$. coli isolates was conducted using disc diffusion method on Mueller-Hinton agar against 25 antibiotics (HiMedia) as per modified Kirby-Bauer Method (Carter et al., 1973). The breakpoint of inhibition zone was interpreted on the basis of zone size interpretation data provided by manufacturer.

\section{Tetracycline resistant gene profiling}

The multiplex polymerase chain reaction (mPCR) was conducted for genotypic characterization of 79 phenotypically tetracycline resistant faecal $E$. coli isolates. The purified isolated colonies of $E$. coli were inoculated into $5 \mathrm{ml}$ Luria broth (LB broth) and incubated for $37^{\circ} \mathrm{C}$ for 24 hours and bacterial DNA was extracted by hot-cold lysis method. The PCR primers as described by Guillame et al., (2000) were used to amplify the tetA) $372 \mathrm{bp}$ and tet(B) $228 \mathrm{bp}$ genes responsible for encoding tetracycline resistance. The mPCR assay was standardized in $25 \mu 1$ reaction mixture and the reaction mixture contained: $10 \mathrm{mM}$ dNTPs $1 \mu 1,25 \mathrm{mM}$

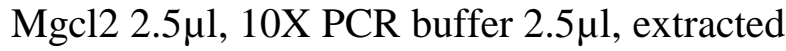
DNA $5 \mu 1$, primer [tet(A) and tet(B)] $1 \mu 1$ each, Taq DNA polymerase $1 \mu 1$ and nuclease free water to make a final volume of $25.0 \mu$. The PCR amplification was conducted using a thermal cycler (BiometraTgradient, Germany) with an initial denaturation at $94^{\circ} \mathrm{C}$ for 5 minutes, thirty-five PCR cycles each of denaturation at $94^{\circ} \mathrm{C}$ for 1 minute, annealing at $50^{\circ} \mathrm{C}$ for 1 minute, extension at $72^{\circ} \mathrm{C}$ for
$45 \mathrm{sec}$ followed by a step of final extension at $72^{\circ} \mathrm{C}$ for 7 minutes. The negative control contained of nuclease free water instead of DNA template while standards of tet (A) and (B) available at Department of Veterinary Public Health, GADVASU, Ludhiana were used as positive control. The PCR products were electrophoretically analysed in $2 \%$ Agarose gelin 1X TBE buffer having ethidium bromide $(0.50 \mu \mathrm{g} / \mathrm{ml})$ at $80 \mathrm{~V}$ for 1 hour. Agarose gels were visualized and photographed in Gel Documentation System (BioRad Pvt Ltd.).

\section{Results and Discussion}

Isolation and serotyping of the $E$. coli isolates

Out of 100 faecal samples, 154 isolates of $E$. coli were confirmed as per cultural and biochemical characterization. All the 154 isolates were got serotyped from Central Research Institute, Kasauli, Himachal Pradesh. Serotyping of 154 E. coli isolates of cattle indicated that $112(72.72 \%)$ isolates were typeable, $28(18.18 \%)$ were untypeable and $14(9.09 \%)$ were rough. The most predominant serotype were found to be $\mathrm{O} 22$ (26 isolates) followed by O56 (7), O60 (5), O120 and $\mathrm{O} 1$ (4 each), O69, O1, O41, O59, O79, O87 and O147 (3 each), O21, O128, O16, O48, O105, O73, O85, O97, O159, O2, O25, O149, O5 and O88 (2 each) and O101, O110, O125, O13, O130, O17, O170, O28, O32, O96, O152, O20, O91, O103, O137, O100, O4 (1 each).

Nearly $19.48 \%$ of isolates are found to be pathogenic for humans like O22, O103, O128 and 091 , whereas nearly $40 \%$ isolates from healthy cattle of German Democratic Republic were of pathogenic serotypes (Montenegro et al., 1990). Various serogroups of faecal E. coli isolated from calves in India include O22, O24, O55, O62, 
O86, O110, O128, O131, O157, O171, O172, O168, O5, O2 and O167 (Arya et al., 2008). In the present study, none of the isolate out of 154 isolates of the bovine faecal sample was found to be of serotype O157. Singh et al., 2007 and Kaur, 2007 also reported the absence of 0157 in cattle in this region of the country. However, Kanwar (1999) found three $\mathrm{O} 157$ serotype out of $50 \mathrm{E}$. coli isolates from calf diarrhoea cases in the same geographical location. The absence of serotype $\mathrm{O} 157$ can be due to the reason that animals may not be shedding this serotype as samples were collected from apparently healthy animals. The various serogroups frequently reported in India with public health signifance are O24(Kaura et al., 1991), O2, O5, O9, O62, O55, O86, O131, O157 and O172 (Wani et al., 2003; Sharma et al., 2004). Serogroups like O2, O55, O86, O128 and $\mathrm{O} 157$ have recurrently been linked with gastrointestinal illness among neonates and adults suffering from haemorrhagic diarrhoea/HC and/or HUS (Nishikawa et al., 2002) which are also of public health concern.

\section{Phenotypic antibiotic resistant profile of the $\mathbf{E}$. coli isolates}

The phenotypic characterization of faecal $E$. coli isolates to determine the drug resistance pattern against 25 antimicrobials resulted in various antimicrobial resistance profiles. The highest resistance was observed against clindamycin and penicillin as $154(100 \%)$ isolates were resistant to both antibiotics, followed by metronidazole 152 (98.70\%), neomycin 146 (94.81\%), kanamycin 139 (90.26\%), cephalothin $133 \quad$ (86.36\%), cephalexin $79(51.30 \%)$, tetracycline 79 (51.30\%), streptomycin 75 (48.70\%), doxycycline $74(48.05 \%)$, trimethoprim 60 (38.96\%) and ampicillin 58 (37.66\%) resistant isolates, and less frequently to the cotrimoxazole 56 (36.36\%), nalidixic acid 54 (35.06\%), polymyxin B 54 (35.06\%), furazolidone 54 (35.06\%), amoxycillin 51 (33.12\%), gentamicin $42 \quad(27.27 \%)$, ciprofloxacin 29 (18.83\%), colistin 28 (18.18\%), enrofloxacin $29 \quad(18.83 \%)$, cefazolin 27 (17.53\%), ceftriaxone 26 $(16.88 \%)$, gatifloxacin $25(16.23 \%)$ and chloramphenicol $15(9.74 \%)$ isolates.

The high resistance against penicillin and metronidazole varying from $96.4-100 \%$ is also observed at other parts of world like Zambia, Malaysia (Radu et al., 2001; Mubita et al., 2008), whereas at Czech dairy farms, $E$. coli isolates exhibited highest resistance to tetracycline followed by resistance to streptomycin (Dolejska et al., 2008). Indian E. coli isolates are usually found to have high resistance against metronidazole $(100 \%)$ and penicillin (98.08\%) antibiotics (Singh et al., 2007; Kaur, 2007) as these are amongst the most commonly used drugs under field conditions for the treatment of animals. The $E$. coli isolates are also frequently resistant to streptomycin $(85.7 \%)$, tetracycline $(80.0 \%)$ as compared to trimethoprim (29.1\%)and nalidixic acid $(29.1 \%$ ) antibiotics (Hoyle et al., 2005). Kanwar (1999) also found E. coli isolates from calf diarrhea resistant to metronidazole $(98.08 \%)$ and co-trimoxazole $(34.62 \%)$ similar to the resistant pattern observed in present study. At eastern part of India, different resistance profile of ampicillin (25.4\%) tetracycline (23.8\%), streptomycin (14.3\%), cephalothin $(86.36 \%)$, cotrimoxazole $(9.5 \%)$, nalidixic acid $(6.4 \%)$ and neomycin $(94.81 \%)$ was observed (Khan et al.,2002)as compared to northern India in the present study viz. $51.30 \%, 48.70 \%, 86.36 \%$, $36.36 \%, 35.06 \%$ and $94.81 \%$ of the E. coli isolates presenting resistance against tetracycline, streptomycin, cephalothin, Cotrimoxazole, nalidixic acid and neomycin, respectively. The commensal E. coli isolated from food-producing animals may exhibit moderate to low level antibiotic resistance i.e. ampicillin (22.5\%), cephalothin (20\%), 
amoxycillin (12.5\%), ceftriaxone (0\%), gentamicin (5\%), kanamycin $(12.5 \%)$, neomycin (15\%), streptomycin (20\%), tetracycline $(70 \%)$, nalidixic acid $(2.5 \%)$ and ciprofloxacin (0\%) as observed by Knezevic and Petrovic (2008)at Serbia. The resistance of the isolates to Ceftriaxone was $16.88 \%$, in contrary to that was reported by Srinivasan $e t$ al., (2007) who reported 3.1\% resistance. The resistance to gentamicin, cephalothin and trimethoprim can be less than $20 \%$ (Srinivasan et al., 2007) or more than $20 \%$ as observed in current study. The resistance of the isolates to tetracycline $(51.30 \%)$ is more in this part of India as compared to other parts where around $25-47 \%$ resistance against tetracycline may be present (Khan et al., 2002; Srinivasan et al., 2007; Gow et al., 2008). However, very high resistance (up to $98 \%$ ) to tetracycline may be present as reported by Donaldson et al., (2006). The resistances of the isolates to chloramphenicol was less $9.74 \%$ as observed earlier also by Radu et al., (2001) and may be due to the fact that chloramphenicol is not frequently used under field conditions for treatment purpose, whereas in country like Pennsylvania, USA, E. coli isolates exhibited very high resistance (98\%) to chloramphenicol (Donaldson et al., 2006; Sawant et al., 2007). Though chloramphenicol is banned from use in food animals, florfenicol, a structural analog of chloramphenicol, approved by the Food and Drug Administration in 1996, is commonly used for treating bovine respiratory pathogens in this part of world.

Comparatively, the high resistance profile of antibiotic resistance against the frequently used antibiotics was detected in the E. coli isolates. Virtually, numerous antimicrobial drugs like penicillins, cephems, tetracycline and fluoroquinolones are being doled out with no control and might be easily obtained without prescription. The abuse and indiscrimination of antimicrobial agents either for curing of animal diseases, feed additive (growth promotion) as well as for preventive measures is not controlled. Therefore, the misuse of antibiotics is frequently associated with an incomplete course of treatment, or sub-therapeutic dose and mistake for drug of choice might be considered the principle causes of high prevalence of antimicrobial resistance. The abuses of antibiotics may lead to high occurrence of resistance amongst $E$. coli strains and enhance the possibility of development of multiple antibiotic resistance (MAR) posing public health risk. The isolation of multiple drug resistant E. coli from dairy farms in this area may make difficulty in success of antimicrobial therapeutic regimen.

The high prevalence of antibiotic resistance patterns was also observed in calves which might be related to specific genes like adhesion genes that represents a high frequency in the micro flora of younger calves and may be found on the same plasmid carrying antimicrobial resistance genes (Dolejska et al., 2008). The high level of resistance in young animals suggest that there is a correlation between resistance, animal age and acquirement of resistance commensal flora (Hoyle et al., 2005; Donaldson et al., 2006; Gow et al., 2008). It can also be attributed to the fact that the younger calves might be more exposed to antibiotic resistant E. coli that spread into the dairies through the wastes, and consequently get picked up and reaches to intestinal tract of young calf, even without therapeutically use (Berge et al., 2005).

\section{Tetracycline resistant gene profiling}

The genotypic characterization of tetracycline resistant tet(A) and tet(B) genes in faecal $E$. coli isolates revealed that the tet(A) gene was predominant gene as was detected in 22 $(27.85 \%)$ isolates as compared to tet(B) gene 
observed in $8(10.26 \%)$ isolates, whereas 15 $(18.99 \%)$ isolates carried both tet(A) and tet(B) gene. Lanz et al., (2003) reported that the tet(A) gene is frequently reported at farms suffering from diarrhoea and enterotoxaemia whereas tet(B) gene was frequently constant prevalent until 1994 (Mayrand et al., 2003); while at beginning of 1995 to 2000 , tet(A) and tet $(\mathrm{C})$ were the most dominant closely associated tetracycline resistance genes. The study conducted on three flocks with diverse histories of antimicrobial application exhibiting comparable results revealed that tet(B) was frequently dominant gene as compared to tet (A) and tet (C) (Lee et al., 1993). Similarly, Sawant et al., (2007) reported that tet(B) was predominant gene (93\%) followed by tet(A) (7\%). The tetracycline resistance $E$. coli may carry the tet(B) gene varying from $60-80 \%$ (Blake et al., 2003; Wilkerson et al., 2004).

Figure.1 Detection of tet(A) and tet(B) genes by multiplex PCR.

Lane A, B and C: tet(A) gene, tet(B) gene and both tet(A) \& (B) gene, respectively; Lane M: 100 bp DNA ladder; Lane +ve: positive control; Lane -ve: negative control

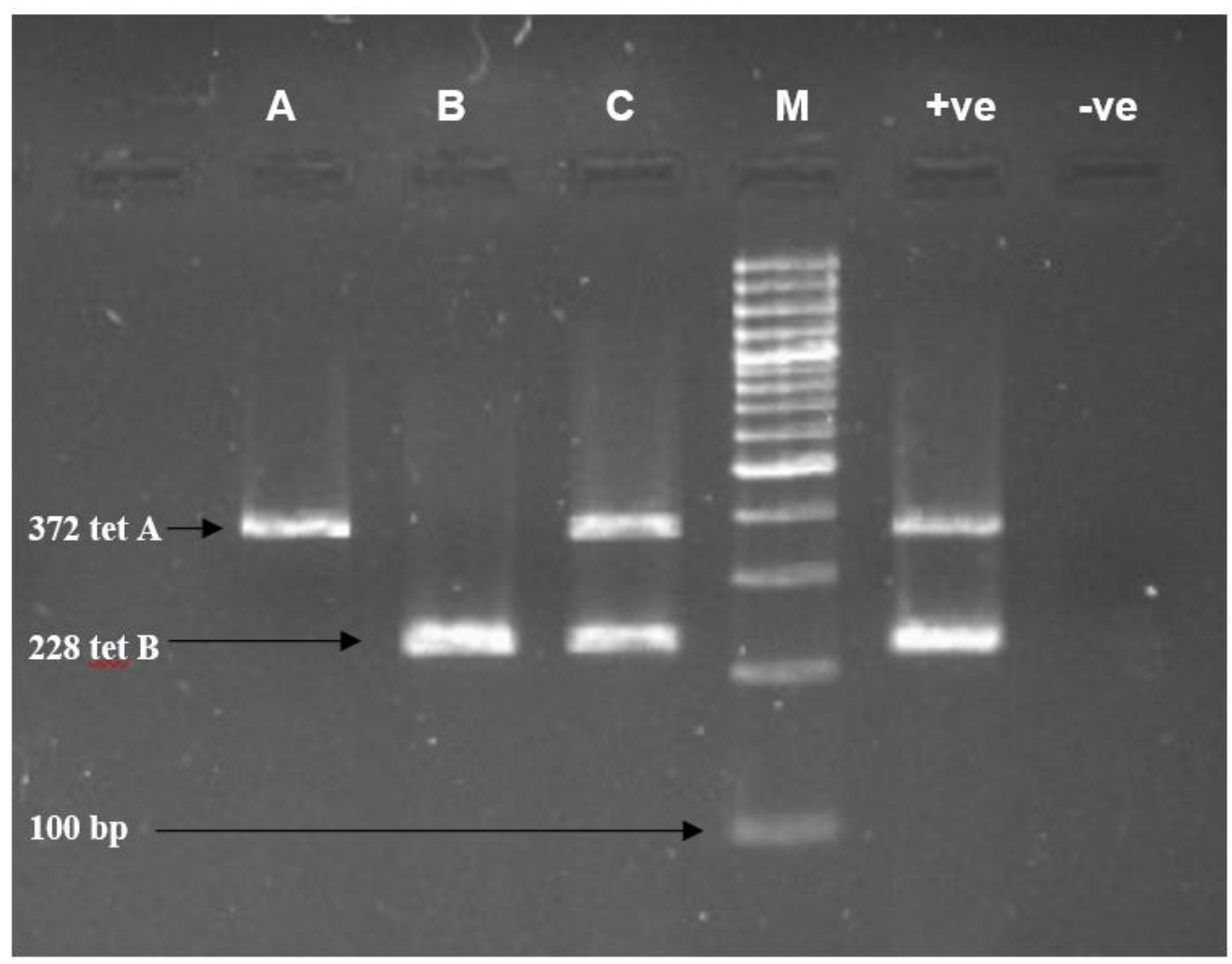

The absence of both tet(A) or tet(B) gene in 34 out of 79 tetracycline resistant E. coli isolates emphasize the need for screening of these isolates for other known tet genes also. The tetracycline resistant $E$. coli isolates may carry unknown tetracycline resistant genes as observed earlier for $E$. coli isolated from humans or other animals (Miranda et al., 2003; Wilkerson et al., 2004). More than one tetracycline resistance genes may be found from the same E. coli isolate (Maynard et al., 2003; Villedieu et al., 2003). Nevertheless, 
this did not have any influence on antibiotics resistance patterns of $E$. coli isolates. The high level resistance profile of tetracycline is not potentially influenced by number of genes (Villedieu et al., 2003). Fairly, this might be as certain of tet genes are enclosed inside conjugative transposon. It's well known that conjugative transposons play a potential role and considered an important determinant for antibiotic resistance. The conjugative transposons are also infrequent in that their relocation activities are synchronised by tetracycline via a complex regulatory network. The carrying of single conjugative transposon may not effect as the same cell being able to receive other correlated or not correlated conjugative transposons (Chopra and Roberts, 2001). This may help E. coli in further dissemination of the genotypic tetracycline resistance as observed in other bacteria like Neisseria, Haemophillus and Streptococcus which are naturally competent to disseminate resistance genes (Roberts, 1998). The presence of tet genes can mobile genetic material like plasmid and transposon helping in the spreading or dissemination of these resistance genes (DePaola and Roberts, 1995).

The results of phenotypic resistance patterns of $E$. coli isolates are mostly influenced by gene determinants. However, the association between phenotypic and genotypic appearance of antibiotic resistance patterns might be influenced by numerous assortment inducing factors such as the usage of particular antibiotic (antibiotic-induced stress responses). Multiple phenotypic antibiotic resistance among $E$. coli isolates in relation to the frequency distribution of resistance genes indicate the presence of multi-gene resistance. These findings highlight the urgent requirement on restriction and regulation of antimicrobial agent in the veterinary practices so as to minimize the risk of development of resistant organisms especially multidrug resistance (MDR).

\section{References}

Arya, G., Roy, A., Choudhary, V., Yadav, M.M. and Joshi, C.G., 2008. Serogroups, atypical biochemical characters, colicinogeny and antibiotic resistance pattern of shiga toxin producing Escherichia coli isolated from diarrhoeic calves in Gujarat, India. Zoonoses Public Health, 55, 89-98.

Authier, S., Paquette, D., Labrecque, O. and Messier, S., 2006. Comparison of susceptibility to antimicrobials of bacteria isolates from companion animals in a veterinary diagnostic laboratory in Canada between 2 time points 10 years apart. Canadian Veterinary Journal, 47, 774-778.

Berge, A.C.B., Atwill, E.R. and Sischo, W.M., 2005. Animal and farm influences on the dynamics of antibiotic resistance in faecal Escherichia coli in young dairy calves. Preventive Veterinary Medicine, 69, 25-38.

Blake, D.P., Humphry, R.W., Scott, K.P., Hillman, K., Fenlon, D.R. and Low, J.C., 2003. Influence of tetracycline exposure on tetracycline resistance and the carriage of tetracycline resistance genes within commensal Escherichia coli populations. Journal of Applied Microbiology, 94, 1087-1097.

Carter, G.R., 1973. Diagnostic procedures in veterinary microbiology $2^{\text {nd }} E d n . p p .46-$ 60 Charles C.Thomas Publisher, Springfield II, USA.

Catry, B., Laevens, H., Devriese, L.A., Opsomer, G., Kruif, A., 2003. Antimicrobial resistance in livestock. $J$. Vet. Pharmacol. Ther, 26, 81-93.

Chopra, I., and Roberts, M., 2001. Tetracycline antibiotics: mode of action, applications, molecular biology and epidemiology of bacterial resistance. Microbiology and Molecular Biology Reviews, 65, 232-260.

DePaola A and Roberts M C., 1995. Class D 
and E tetracycline resistance determinants in gram negative catfish pond bacteria. Molecular and Cellular Probes, 9: 311-313.

Dolejska, M., Senk, D., Cizek, A., Rybarikova, J., Sychra, O. and Literak, I., 2008. Antimicrobial resistant Escherichia coli isolates in cattle and house sparrows on two Czech dairy farms. Research in Veterinary Science, 85, 491-494.

Donaldson, S.C., Straley, B.A., Hegde, N.V., Sawant, A.A., DebRoy, C. and Jayarao, B.M., 2006. Molecular epidemiology of ceftiofur-resistant Escherichia coli isolates from dairy calves. Applied and Environmental Microbiology, 72, 3940 3948.

Gellin, G., Langlois, B., Dawson, K.A. and Aaron, D.K., 1989. Antibiotic resistance of gram negative enteric bacteria from pigs in three herds with different histories of antibiotic exposure. Applied and Environmental Microbiology, 55, 2287-2292.

Gow, S. P., Waldner, C. L., Rajic, A., McFall, M. E. and Reid-Smith, R., 2008. Prevalence of antimicrobial resistance in faecal generic Escherichia coli isolated in western Canadian cow-calf herds Part I -Beef calves. Canadian Journal of Veterinary Research, 72, 8290.

Guillame, G., Verbrugge, D., ChasseurLibotte, M.L., Moens, W. and Collard, J.M., 2000. PCR typing of tetracycline resistance determinants (tet A-E) in Salmonella enterica serotype Hadar and in the microbial community of activated sludge from hospital and urban wastewater treatment facilities in Belgium. Federation of European Microbiological Societies Microbiology Letters, 32, 77-85.

Hoyle, D.V., Yates, C.M., Chase-Topping, M.E., Turner, E.J., Davies, S.E., Low,
J.C., Gunn, G.J., Woolhouse, M.E. and Amyes, S.G.B., 2005. Molecular epidemiology of antimicrobial-Resistant commensal Escherichia coli strains in a cohort of newborn calves. Applied and Environmental Microbiology, 71, 66806688.

Kanwar, R., 1999. Studies on the bacterial agents implicated in calf diarrhea with special reference to Escherichia coli and Salmonella spp. M.V.Sc. thesis, College of Veterinary Science, Punjab Agricultural University, Ludhiana pp. 47-61.

Kaur, A., 2007. Epidemiological studies on Vero toxin-producing Escherichia coli from faeces of healthy dairy cattle and buffalo. M.V.Sc thesis, College of Veterinary Science, Punjab Agricultural University, Ludhiana.

Kaura, Y.K., Minakshi, K.T. and Chaturvedi, G.C., 1991. Characteristics of E. coli strains isolated from diarrhoeic buffalo and cow calves. Indian Journal of Microbiology, 31, 33-47.

Khan, A., Dass, S. C., Ramamurthy, T., Sikdar, A., Khanam, J., Yamasaki, S., Takida, Y. and Nair, G. B., 2002. Antibiotic resistance, virulence genes and molecular profiles of shiga-toxin producing Escherichia coli isolates from diverse sources in Calcutta. Indian Journal of Clinical Microbiology, 40, 2009-2015.

Knezevic, P. and Petrovic, O., 2008. Antibiotic resistance of commensal Escherichia coli of food-producing animals from three Vojvodinian farms, Serbia. International Journal of Antimicrobial Agents, 31, 360-363.

Langlois, B.E., Dawson, K.A., Leak, I. and Aaron, D.K., 1988. Antimicrobial resistance of faecal coliforms from pigs in a herd not exposed to antimicrobial agents for 126 months. Veterinary Microbiology, 18, 147-153. 
Lanz, R., Kuhnert, P. and Boerlin, P., 2003. Antimicrobial resistance and resistance gene determinants in clinical Escherichia coli from different animal species in Switzerland. Veterinary Microbiology, 91, 73-84.

Lee, C., Langlois, B.E. and Dawson, K.A., 1993. Detection of tetracycline resistance determinants in pig isolates from three herds with different histories of antimicrobial agent exposure. Applied and Environmental Microbiology, 59, 1467-1472.

Maynard C, Fairbrothe J.M. Bekal S, Sanschagrin F, Levesque R.C, Brousseau R, Masson L, Lariviere S and Harel J., 2003. Antimicrobial resistance genes in enterotoxigenic Escherichia coli O149:K91 isolates obtained over a 23-year period from pigs. Antimicrobial Agents Chemotherapy, 47: 3214-3221

Miranda, C.D., Kechrenberg, C., Ulep, C., Schwarz, S. and Roberts, M.C., 2003. Diversity of tetracycline resistance in bacteria from Chilean Salmon farm. Antimicrobial Agents and Chemotherapy, 47, 883-888.

Montenegro, M.A., Bulte, M., Trumpf, T., Aleksic, S., Reuter, G., Bulling, E. and Helmulth, R., 1990. Detection and characterization of faecal verotoxinproducing Escherichia coli from healthy cattle. Journal Clinical Microbiology 28, 1417-1421.

Moyaert, H., De Graef, E.M., Haesebrouck, F. and Decostere, A., 2006. Acquired antimicrobial resistance in the intestinal microbiota of diverse cat populations. Research in Veterinary Science, 81, 1-7. Mubita, C., Syakalima, M., Chisenga, C., Bwalya, M., Chifumpa, G., Hangombe, B.M., Sinkala, P., Simuunza, M., Fukushi, H., Isogai, H., Yasuda, J. and Isogai, E., 2008. Antibiograms of faecal Escherichia coli and Enterococci species isolated from pastoralist cattle in the interface areas of the Kafue basin in Zambia - short communication. Veterinarski Arhiv, 78, 179-185.

Nishikawa, Y., Zhou, Z., Hase, A., Ogasawara, J., Kitase, T., Abe, N., Nakamura, H., Wada, T., Ishii, E. and Haruki, K., 2002. Diarrheagenic Escherichia coli isolated from stools of sporadic cases of diarrheal illness in Osaka city, Japan between 1997 and 2000: prevalence of enteroaggregative E. coli heat stable enterotoxin 1 genepossessing E. coli. Japan Journal of Infectious Disease, 55, 182-190.

Quinn, P.J., Carter, M.E., Morkey, B. and Carter, G.R., 1994. Clinical veterinary microbiology. pp. 209-225. Mosby Year Book Europe Ltd., London.

Radu, S., Ling, O.W., Rusul, G., Abdul Karim, I.M. and Nishibuchi, M., 2001. Detection of Escherichia coli O157:H7 by multiplex PCR and their characterization by plasmid profiling, antimicrobial resistance, RAPD and PFGE analyses. Journal of Microbiology Methods, 46, 131-139.

Roberts M C., 1998. Antibiotic resistance in oral/respiratory bacteria. Critical Reviews in Oral Biology and Medicine, 9: 522-540.

Rosas, I., Salinas, E., Martınez, L., Calva, E., Cravioto, A., Eslava, C. and AmabileCuevas, C.F., 2006. Urban dust faecal pollution in Mexico City: antibiotic resistance and virulence factors of Escherichia coli. International Journal of Hygiene and Environmental Health, 209, 461-470.

Sawant, A.A., Hegde, N.V., Straley, B.A., Donaldson, S.C., Love, B.C., Knabel, S.J. and Jayarao, B.M., 2007. Antimicrobial resistant enteric bacteria from dairy cattle. Applied and Environmental Microbiology, 73, 156163.

Sharma, D.K., Soni, S.S., Kashyap, S.K. and 
Shringi, B.N., 2004. Seroprevalence, antibiotic sensitivity pattern and transfer of plasmid coded characters of E. coli. Indian Veterinary Journal, 81, 6-8.

Singh, M. Singh, R. Arora, Anil and Sandhu, K. S., 2007. Molecular characterization of Escherichia coli isolated from diarrhoeic neonatal calves. Indian Journal of Animal Sciences, 77(11):1063-1068.

Sorum, H. and Sunde, M., 2001. Resistance to antibiotics in the normal flora of animals. Veterinary Research, 32, 227241.

Srinivasan, V., Gillespie, B.E., Lewis, M.J., Nguyen, L.T., Headrick, S.I., Schukken, Y.H. and Oliver, S.P., 2007. Phenotypic and genotypic antimicrobial resistance patterns of Escherichia coli isolated from dairy cows with mastitis Veterianry Microbiology, 124, 319-328.
McNab, R. and Spratt, D.A., 2003. Prevalence of tetracycline resistance genes in oral bacteria. Antimicrobial agents and Chemotherapy, 47, 878-882.

Wani, S.A., Bhatt, M.A., Samanta, I., Nishikawa, Y. and Buchh, A.S., 2003. Isolation and characterization of shigatoxin producing Escherichia coli (STEC) and enteropathogenic Escherichia coli (EPEC) from calves and lambs with induce shiga toxinencoding bacteriophages, toxin diarrhoea in India. Letters in Applied Microbiology, 37, 121-126.

Wilkerson, C., Samadpour, M., Kirk, N.W. and Roberts, M.C., 2004. Antibiotic resistance and distribution of tetracycline resistance genes in Escherichia coli $\mathrm{O} 157: \mathrm{H} 7$ isolates from humans and bovines. Antimicrobials Agents Chemotherapy, 48, 1066-1067.

\section{How to cite this article:}

Mahmoud, A. S., D. Mittal, G. Filia, V. Ramneek and Mahajan, V. 2020. Prevalence of Antimicrobial Resistance Patterns of Escherichia coli Faecal Isolates of Cattle. Int.J.Curr.Microbiol.App.Sci. 9(03): 1850-1859. doi: https://doi.org/10.20546/ijcmas.2020.903.214 